\title{
Poly (ADP-ribose) polymerase 1 transcriptional regulation: A novel crosstalk between histone modification H3K9ac and ETS1 motif hypomethylation in BRCA1-mutated ovarian cancer
}

\author{
Da Li ${ }^{1}$, Fang-Fang Biं ${ }^{1}$, Ji-Min $\mathrm{Cao}^{2}$, Chen $\mathrm{Cao}^{3}$, Chun-Yan Li ${ }^{4}$, Bo Liu ${ }^{5}$, Qing Yang ${ }^{1}$ \\ ${ }^{1}$ Department of Obstetrics and Gynecology, Shengjing Hospital of China Medical University, Shenyang, China \\ ${ }^{2}$ Department of Physiology and Pathophysiology, Institute of Basic Medical Sciences, Chinese Academy of Medical Sciences, \\ School of Basic Medicine Peking Union Medical College, Beijing, China \\ 3 Department of Pathology, Chinese PLA General Hospital, Beijing, China \\ ${ }^{4}$ Department of Histology and Embryology, Institute of Basic Medical Sciences, Chinese Academy of Medical Sciences, School \\ of Basic Medicine Peking Union Medical College, Beijing, China \\ ${ }^{5}$ Department of Laboratory Medicine, No. 1 Hospital of China Medical University, Shenyang, China \\ Correspondence to: Da Li and Qing Yang, email: leeda@ymail.com
}

Keywords: PARP1, H3K9ac, ETS1, BRCA1, Ovarian cancer

Received: October 29, $2013 \quad$ Accepted: December 29, $2013 \quad$ Published: December 29, 2013

This is an open-access article distributed under the terms of the Creative Commons Attribution License, which permits unrestricted use, distribution, and reproduction in any medium, provided the original author and source are credited.

\section{ABSTRACT:}

Poly (ADP-ribose) polymerase 1 (PARP1) plays a critical role in ovarian cancer progression. However, the epigenetic mechanism regulating PARP1 transcription remains largely unknown. Here, we show that the hypomethylated ETS1 motif is a key regulatory element for the PARP1 gene in BRCA1-mutated ovarian cancer. Mechanistically, the ETS1 motif hypomethylation-mediated increase of active histone marker H3K9ac and transcription factor ETS1 enrichment synergistically activates PARP1 transcription. Clinicopathological data indicate that a hypomethylated ETS1 motif was associated with high-grade tumors $(P=0.026)$ and $p N 1(P=$ 0.002 ). Univariate survival analysis demonstrated an association between the hypomethylated ETS1 motif and an increased risk of death in BRCA1-mutated ovarian cancer patients. Our findings imply that the genetic (such as BRCA1 mutation) and epigenetic mechanisms (such as hypomethylated ETS1 motif, and histone modification H3K9ac and transcription factor ETS1 binding) are jointly involved in the malignant progression of PARP1-related ovarian cancer.

\section{INTRODUCTION}

Ovarian cancer is characterized by a high mortality rate among gynecologic malignancy worldwide [1]. Accumulating evidence indicates that hereditary factors (e.g., BRCA1) [2] and epigenetic events [3,4] are involved in the initiation and progression of ovarian cancer. However, the joint effect of genetic and epigenetic mechanisms has not been extensively studied. In 2005, two pivotal studies showed that BRCA-deficient cells were especially sensitive to chemical inhibitors of poly (ADP-ribose) polymerase (PARP), which plays a critical role in single-stranded DNA break repair, presumably due to the lack of homologous recombination-dependent DNA repair $[5,6]$. These findings have raised significant concerns about PARP in BRCA-deficient ovarian cancer.
Our previous results suggested that hypomethylation of the promoter region, especially around the ETS1 motif may be responsible for PARP1 overexpression in BRCA1-mutated serous ovarian cancer [7]. However, the exact mechanism of abnormal promoter methylationrelated PARP1 expression is still not entirely clear. Therefore, the present study was undertaken to investigate PARP1 transcriptional regulation from genetic (BRCA1 mutation) and epigenetic (promoter methylation, histone modifications and transcription factor binding) aspects, and to provide novel insight into epigenetic changemediated abnormal PARP1 expression in BRCA1-mutated ovarian cancer progression. 


\section{RESULTS}

\section{Hypomethylated ETS1 motif is a key regulatory mechanism for PARP1 transcription in BRCA1- mutated ovarian cancer}

Our previous results suggested that promoter hypomethylation, especially around the ETS1 and ETS2 motifs, may be involved in the up-regulation of PARP1 expression in BRCA1-mutated ovarian cancer. To further confirm the role of the cytosine located at the ETS1 and ETS2 motifs, a point mutation of cytosine to thymine was constructed (Fig. 1A). Notably, the ETS1 motif was the critical site for PARP1 transcription only in BRCA1-mutated ovarian cancer cells (Fig. 1Bi-Biv). Recently, a substantial body of evidence suggests that most of the known genes contain specific motifs, such as G-quadruplex in their promoter regions, which can modulate gene transcription by affecting the binding of histone [8] Therefore, CD spectra were used to gain information on whether methylation of the ETS1 motif can influence the structure of the PARP1 promoter. However, our results showed that ETS1 motif methylation may not affect the chemical structure of the core promoter of PARP1 (Fig. 1C).

\section{Increase of H3K9ac and ETS1 factor enrichment around the hypomethylated ETS1 motif in BRCA1-mutated ovarian cancer}

To obtain further understanding of the regulatory potential of the crosstalk between DNA methylation and histone modification in controlling PARP1 transcription, we examined the active histone markers H3K9ac, H3K18ac, H3K27ac, H3K4me1, H3K4me2, H3K4me3, H3K36me 3 and H3K79me, and the repressive histone markers $\mathrm{H} 3 \mathrm{~K} 9 \mathrm{me}, \mathrm{H} 3 \mathrm{~K} 9 \mathrm{me} 2, \mathrm{H} 3 \mathrm{~K} 9 \mathrm{me} 3, \mathrm{H} 3 \mathrm{~K} 27 \mathrm{me}$, $\mathrm{H} 3 \mathrm{~K} 27 \mathrm{me} 2$ and $\mathrm{H} 3 \mathrm{~K} 27 \mathrm{me} 3$ in the core promoter of PARP1, especially at the ETS1 motif; we also focused on the enrichment of the transcription factor ETS1, due to the fact that the hypomethylated ETS1 motif was observed. Chromatin immunoprecipitation analysis indicated that the levels of H3K9ac and ETS1 factors were significantly increased in BRCA1-mutated ovarian cancer tissue (Fig. 2Ai and Aii). The transcription factor ETS1 and chromatin-modifying enzymes that facilitate the creation of histone modification H3K9ac (GCN5 and PCAF) were also analyzed. Although there was no significant change in the expression of PCAF, the expression levels of GCN5 and ETS1 factors were increased in BRCA1-mutated ovarian cancer (Fig. 2B).
H3K9ac and ETS1 factor present in the ETS1 motif are responsible for the transcriptional regulation of PARP1 in BRCA1-mutated ovarian cancer

We observed that only the combined knockdown of GCN5 and PCAF was able to specifically induce a decrease of H3K9ac around the ETS1 motif (Fig. 3Ci). Interestingly, knockdown of the transcription factor ETS1 showed a similar effect to the combined knockdown of GCN5 and PCAF (Fig. 3Ci). Therefore, an alternative possibility may be that ETS1 factors can recruit GCN5 and PCAF to the ETS1 motif, which is involved in the acetylation of H3K9 (Fig. 3D). As shown in Fig. 3Cii, knockdown of ETS1 factors was an effective way to reduce ETS1 enrichment, but did not change the levels
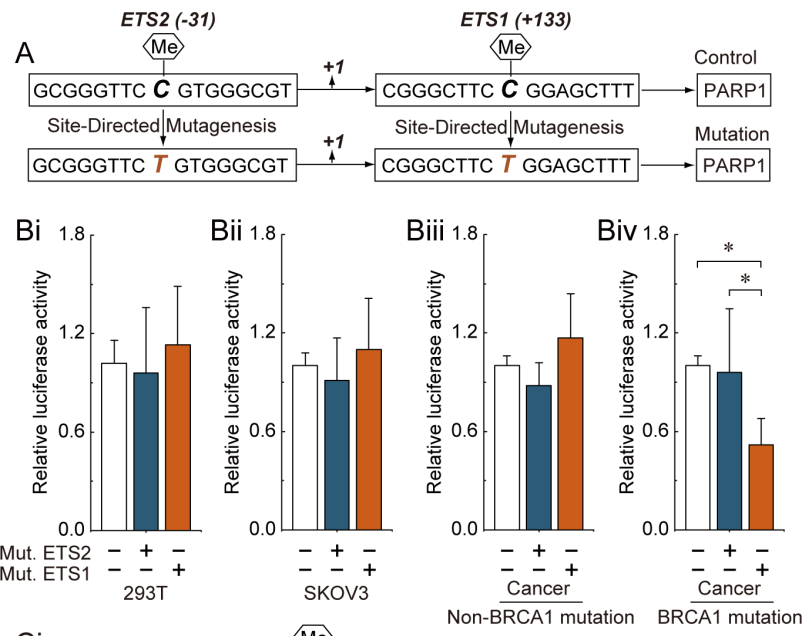

Ci

- chtggcgtcgggcttc C gagctttggcggcagcta

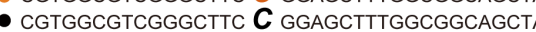

Cii

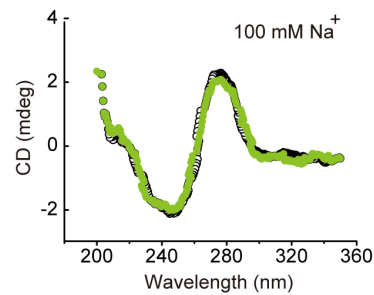

Ciii

Fig 1: ETS1 motif methylation and PARP1 transcriptional activity. (A) the schematic represents the cytosine located at the ETS1 and ETS2 motif that were point mutated to generate the thymine. (Bi-Biv) 293T cells, SKOV3 cells and primary non-mutated and BRCA1-mutated ovarian cancer cells were transfected with mutant plasmids. At 24 hours after transfection, whole-cell extracts were analyzed for luciferase activity. Bar graphs show mean $\pm \mathrm{SD}$. ${ }^{*} \mathrm{P}<0.05$ vs. Control. Mut., Mutation. (Ci) the schematic represents the selected nucleotide sequence with or without a methyl group at the fifth position of the cytosine pyrimidine ring at the ETS1 motif. (Cii) the CD spectra of the selected nucleotide sequence in the presence of $100 \mathrm{mM} \mathrm{Na}+$ or $100 \mathrm{mM} \mathrm{K}+$ are shown. 
of $\mathrm{H} 3 \mathrm{~K} 9 \mathrm{ac}$ around the ETS1 motif. Meanwhile, we observed that knockdown of GCN5, PCAF and ETS1 factors (Fig. 2Ai and Aii) had no detectable effect on the cell morphology and proliferation (Fig. 2Bi and Bii). Notably, we observed that H3K9ac deletion and/or ETS1 enrichment were effective ways to induce a decrease of PARP1 levels in SKOV3 cells and primary non-mutated and BRCA1-mutated ovarian cancer cells (Fig. 3EiEiv). However, the most significant trend was primarily observed in cells originating from BRCA1-mutated ovarian cancer.

\section{Correlation of ETS1 motif methylation with clinicopathological characteristics in BRCA1- mutated ovarian cancer}

As shown in Table 1, the hypomethylated ETS1 motif was associated with high-grade tumor $(\mathrm{P}=0.026)$ and $\mathrm{pN} 1(\mathrm{P}=0.002)$. Moreover, ovarian cancer patients with high levels of CA125 $(\mathrm{P}=0.190)$ and high production of ascites $(\mathrm{P}=0.077)$ showed a trend association with ETS1 motif hypomethylation, although this was not statistically significant.

\section{Multivariate and univariate analysis of overall survival for patients with BRCA1-mutated ovarian cancer}

We analyzed the overall survival to assess the prognostic significance. Multivariate Cox regression analysis indicated that histological grade (Table 2, $\mathrm{P}=$ 0.001), pT (Table 2, $\mathrm{P}=0.018$ ), pM (Table 2, $\mathrm{P}=0.001$ ) and p53 status (Table 2, $\mathrm{P}=0.007$ ) were independent prognostic factors for predicting the overall survival of BRCA1-mutated ovarian cancer patients. We also performed Kaplan-Meier analysis and log-rank tests for overall survival to define prognostic subgroups. The results revealed that the significant prognostic factors (all $\mathrm{P}<0.001$ ) were pN (Fig. 4E), pM (Fig. 4F), FIGO (Fig. 4G), ascites (Fig. 4I) and residual tumor (Fig. 4J). Moreover, patients aged $>60$ years (Fig. 4A), those who were postmenopausal (Fig. 4B), and those with Grade III (Fig. 4C), pT3 (Fig. 4D), CA125 (Fig. 4H), and ETS1 motif methylation (Fig. 4L) ovarian cancer showed a significant trend for poor overall survival (all $\mathrm{P}<0.05$ ). No significant difference in overall survival was found among patients with different p53 status (Fig. 4K).

\section{DISCUSSION}

Promoter methylation, with concurrent changes in histone modifications, is an epigenetic phenomenon which can affect the conformation of chromatin and the accessibility of DNA for transcription factors [9,10]. ETS is one of the largest transcription factor families and has a highly conserved DNA-binding domain that recognizes a common sequence motif, 5'-(C/A) GGA (A/T) -3' [11], which is widely distributed in the PARP1 promoter [12]. In this study, we report for the first time that (i) ETS1 motif methylation is a key regulatory element for PARP1 transcription, (ii) hypomethylated ETS1-mediated

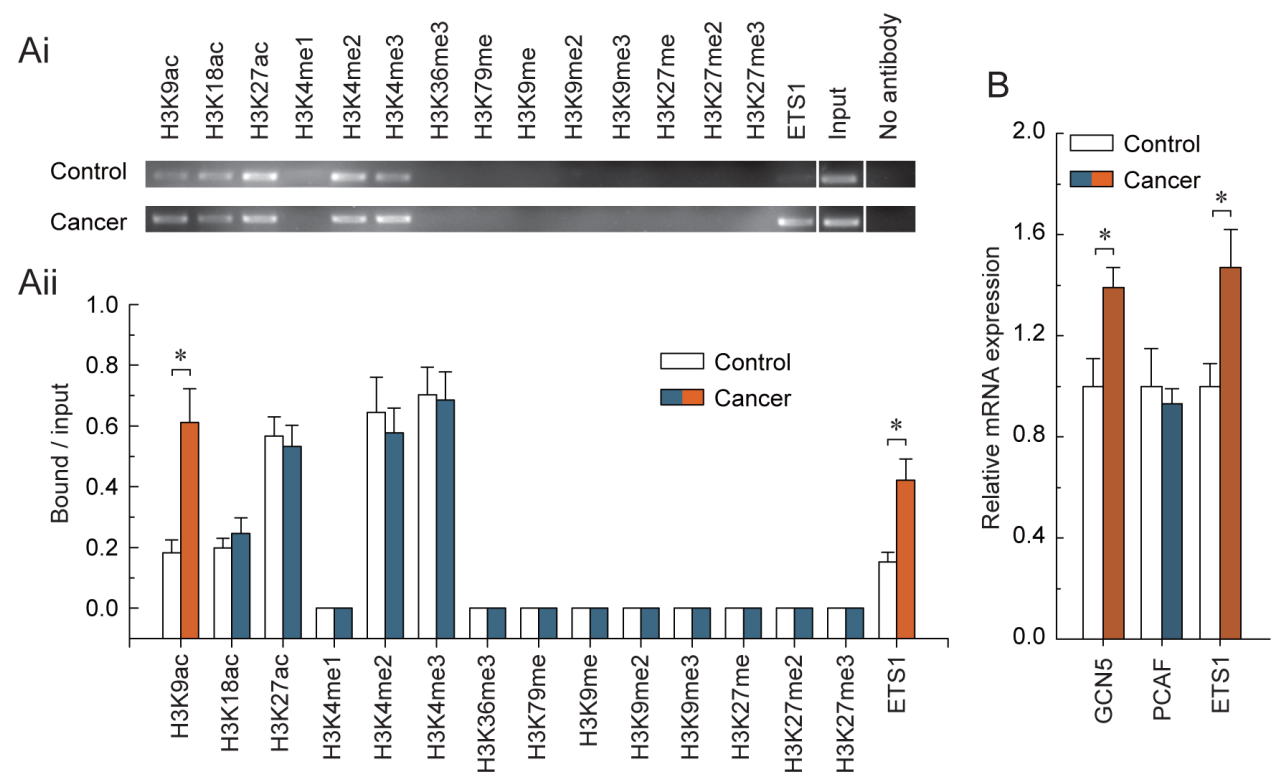

Fig 2: Characteristic histone modification and ETS1 factors enrichment patterns around the hypomethylated ETS1 motif in BRCA1-mutated ovarian cancer. (Ai) chromatin immunoprecipitation was performed using antibodies to H3K9ac, H3K18ac, H3K27ac, H3K4me1, H3K4me2, H3K4me3, H3K36me3, H3K79me, H3K9me, H3K9me2, H3K9me3, H3K27me, H3K27me2, and H3K27me3. PCR was performed for regions around the ETS1 motif. A negative control without antibodies was included for comparison. (Aii) representative results of three independent experiments are shown. (B) expression levels of the GCN5, PCAF and ETS1 factors in BRCA1-mutated ovarian cancer. Bar graphs show mean $\pm \mathrm{SD}, * \mathrm{P}<0.05$ vs. Control. 
increase of histone modification $\mathrm{H} 3 \mathrm{~K} 9 \mathrm{ac}$ and transcription factor ETS1 enrichment synergistically activate PARP1 expression in BRCA1-mutated ovarian cancer, and (iii) the deletion of $\mathrm{H} 3 \mathrm{~K} 9 \mathrm{ac}$ and/or ETS1 enrichment were effective ways to induce a decrease of PARP1 levels in ovarian cancer cells. Moreover, Supplementary Figure 1 shows that BRCA1 status may not directly affect H3K9ac and ETS1 enrichment around the ETS1 motif. Therefore, these results suggest that abnormal hypomethylated ETS1
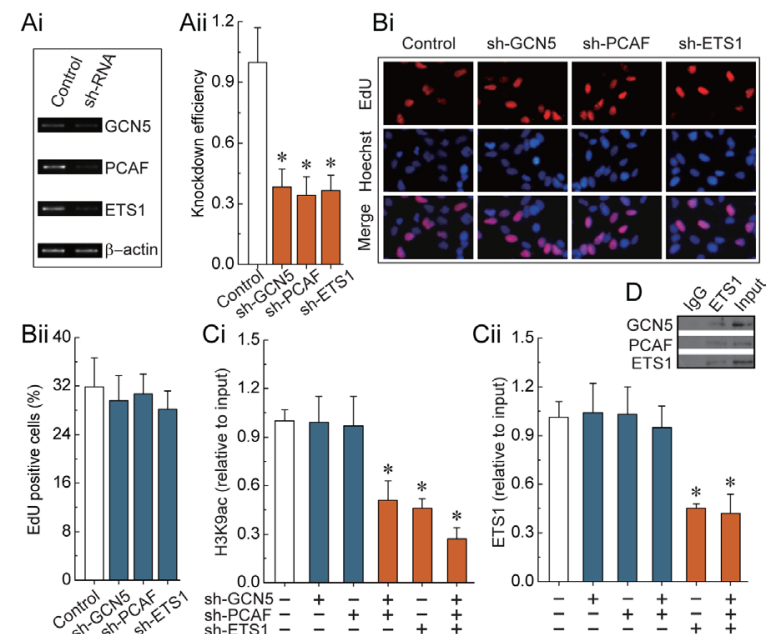

ETS
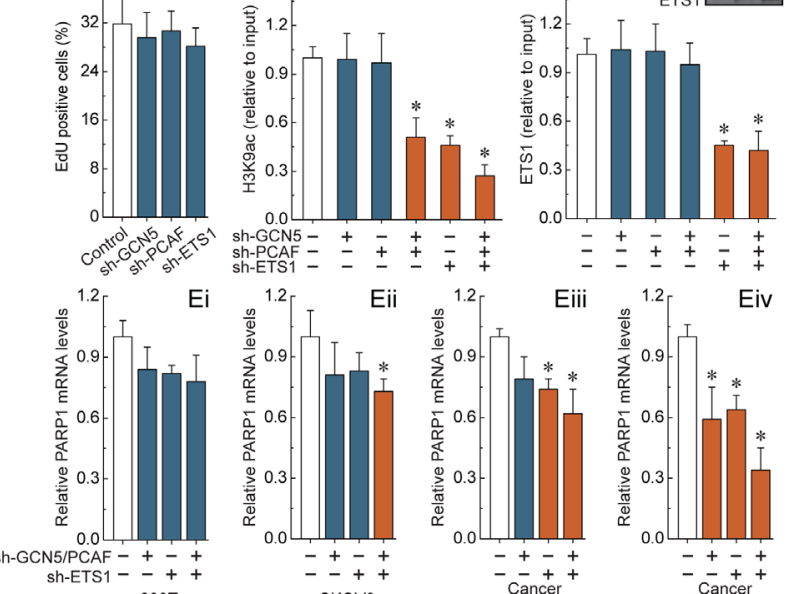

293T

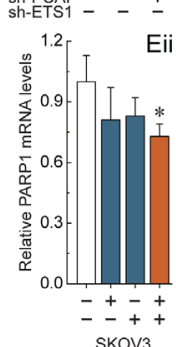

SKOV 3

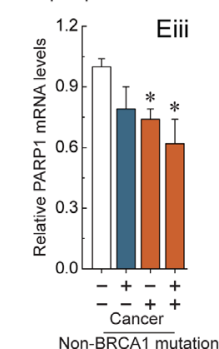

Non-BRCA1 mutation
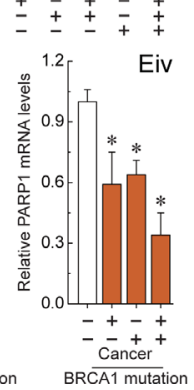

Fig 3: H3K9ac and ETS1-mediated transcriptional regulation of PARP1. (Ai) RT-PCR showing GCN5, PCAF and ETS1 factors levels before and after knockdown by shRNA, and normalized to $\beta$-actin expression. (Aii) representative results of three independent experiments are shown. (Bi) EdU labeling showing proliferation of GCN5, PCAF and ETS1silenced and control cells. Blue, Hoechst 33342 labeling of cell nuclei; Red, EdU labeling of nuclei of proliferative cells. (Bii) the EdU incorporation rate was expressed as the ratio of EdU positive cells to total Hoechst33342 positive cells. (Ci and Cii) analysis of histone modification $\mathrm{H} 3 \mathrm{~K} 9 \mathrm{ac}$ and transcription factor ETS1 enrichment around the ETS1 motif after the deletion of GCN5, PCAF or ETS1 factors. (D) The interactions of ETS1 and GCN5 or PCAF were examined by the immunoprecipitation of cell extracts with an antibody to ETS1, and the coimmunoprecipitation of ETS1, GCN5 and PCAF by western blot analysis. Results of Fig.3 A-D were obtained in BRCA1-mutated ovarian cancer cells, and the same results were also obtained in $293 \mathrm{~T}$ cells, SKOV3 cells, and non-BRCA1 mutated ovarian cancer cells (data not shown). (Ei-Ev) the PARP1 expression levels after deletion of H3K9ac and ETS1 enrichment around the ETS1 motif in 293T cells, SKOV3 cells, and primary nonmutated and BRCA1-mutated ovarian cancer cells. Bar graphs show mean $\pm \mathrm{SD} .{ }^{*} \mathrm{P}<0.05$ vs. Control.
Table 1: Association between PARP1 promoter methylation and clinicopathological features of BRCA1-mutated ovarian cancer

\begin{tabular}{|l|l|l|l|l|l|l|}
\hline & $\mathrm{n}$ & $\mathrm{M}$ & $(\%)$ & $\mathrm{UM}$ & $(\%)$ & $\mathrm{P}$ \\
\hline Age at diagnosis & & & & & & 0.618 \\
\hline$\leq 60$ y & 40 & 15 & 62.50 & 25 & 55.56 & \\
\hline$>60$ y & 29 & 9 & 37.50 & 20 & 44.44 & \\
\hline Menstrual status & & & & & & 0.761 \\
\hline Premenopausal & 15 & 6 & 25.00 & 9 & 20.00 & \\
\hline Postmenopausal & 54 & 18 & 75.00 & 36 & 80.00 & \\
\hline $\begin{array}{l}\text { Histological } \\
\text { grade }\end{array}$ & & & & & & 0.026 \\
\hline I-II & & & & & & \\
\hline III & 33 & 16 & 66.67 & 17 & 37.78 & \\
\hline pT & 36 & 8 & 33.33 & 28 & 62.22 & \\
\hline pT1-2 & 31 & 9 & 37.50 & 22 & 48.89 & \\
\hline pT3 & 38 & 15 & 62.50 & 23 & 51.11 & \\
\hline pN & & & & & & 0.002 \\
\hline pN0 & 47 & 22 & 91.67 & 25 & 55.56 & \\
\hline pN1 & 22 & 2 & 8.33 & 20 & 44.44 & \\
\hline pM & & & & & & 1.000 \\
\hline pM0 & 62 & 22 & 91.67 & 40 & 88.89 & \\
\hline pM1 & 7 & 2 & 8.33 & 5 & 11.11 & \\
\hline FIGO stage & & & & & & 0.794 \\
\hline I/II & 24 & 9 & 37.50 & 15 & 33.33 & \\
\hline III/IV & 45 & 15 & 62.50 & 30 & 66.67 & \\
\hline CA125 & & & & & & 0.190 \\
\hline$\leq 200 U / m l$ & 11 & 45.83 & 13 & 28.89 & \\
\hline$>200 U / m l$ & 45 & 13 & 54.17 & 32 & 71.11 & \\
\hline Ascites & & & & & & 0.077 \\
\hline$\leq 500$ ml & 35 & 16 & 66.67 & 19 & 42.22 & \\
\hline$>500$ ml & 34 & 8 & 33.33 & 26 & 57.78 & \\
\hline Residual tumor & & & & & & 0.615 \\
\hline$\leq 2$ cm & 31 & 12 & 50.00 & 19 & 42.22 & \\
\hline$>2$ cm & & & & & & 0.587 \\
\hline p53 status & & & & & \\
\hline Negative & Positive & & & & \\
\hline
\end{tabular}

FIGO, International Federation of Gynecology and Obstetrics; M, methylated; UM, unmethylated.

motif may be the initial factor, but that PARP1 expression is likely to be the result of a complex interaction of multiple factors in a BRCA1-deficient environment, such as increasing the chromatin-modifying enzyme GCN5 levels and transcription factor ETS1 levels. This observation is consistent with previous reports that ETS transcription factors may be key mediators in regulating PARP expression [12]. Furthermore, an increasing amount of evidence suggests that ETS transcription factors are important regulators of the tumorigenic properties of ovarian cancer cells [13] and correlate with poor survival in serous ovarian carcinoma [14]. 
Table 2: Prognostic factors for overall survival of BRCA1-mutated ovarian cancer patients by multivariate Cox regression analysis

\begin{tabular}{|l|l|l|l|}
\hline Variable & RR & $95 \%$ CI & P \\
\hline $\begin{array}{l}\text { Age at diagnosis ( }>60 \\
\text { y vs } \leq 60 \text { y) }\end{array}$ & 0.979 & $0.934-1.026$ & 0.376 \\
\hline $\begin{array}{l}\text { Menstrual status (Post } \\
\text { vs Pre) }\end{array}$ & 2.938 & $0.643-13.422$ & 0.164 \\
\hline $\begin{array}{l}\text { Histological grade (III } \\
\text { vs I-II) }\end{array}$ & 6.536 & $2.167-19.714$ & 0.001 \\
\hline pT (pT3 vs pT1-2) & 3.196 & $1.222-8.362$ & 0.018 \\
\hline pN (pN1 vs pN0) & 2.677 & $0.703-10.190$ & 0.149 \\
\hline pM (pM1 vs pM0) & 11.954 & $2.847-50.196$ & 0.001 \\
\hline $\begin{array}{l}\text { FIGO stage (III-IV vs } \\
\text { I-II) }\end{array}$ & 1.287 & $0.181-9.153$ & 0.801 \\
\hline $\begin{array}{l}\text { CA125 ( }>200 \text { U/ml vs } \\
\leq 200 \text { U/ml) }\end{array}$ & 0.735 & $0.221-2.444$ & 0.616 \\
\hline $\begin{array}{l}\text { Ascites ( }>500 \mathrm{ml} \text { vs } \leq \\
500 \text { ml) }\end{array}$ & 2.221 & $0.685-7.203$ & 0.184 \\
\hline $\begin{array}{l}\text { Residual tumor ( }>2 \\
\text { cm vs } \leq 2 \text { cm) }\end{array}$ & 1.529 & $0.579-4.039$ & 0.392 \\
\hline $\begin{array}{l}\text { p53 status (Pos vs } \\
\text { Neg) }\end{array}$ & 0.255 & $0.095-0.683$ & 0.007 \\
\hline $\begin{array}{l}\text { Methylation (M vs } \\
\text { UM) }\end{array}$ & 0.540 & $0.164-1.778$ & 0.311 \\
\hline
\end{tabular}

Post, postmenopausal; Pre, premenopausal; FIGO, International Federation of Gynecology and Obstetrics; Pos, positive; Neg, negative; M, methylated; UM, unmethylated; RR, relative risk; CI, confidence interval.
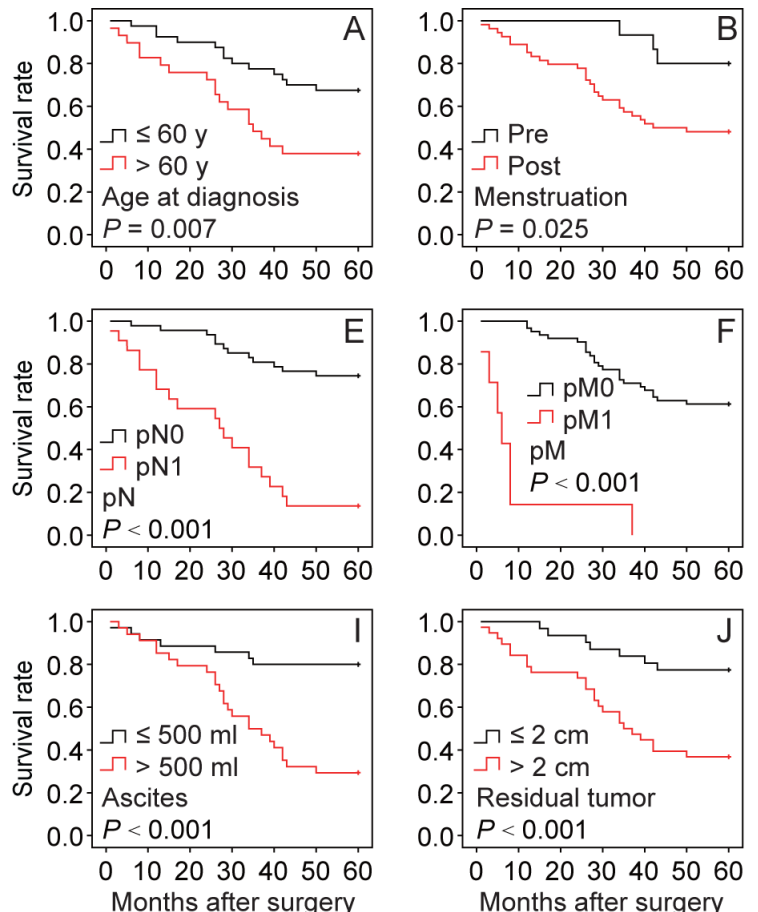

Abnormal promoter methylation is often associated with adverse clinical factors [15]. In line with this, clinicopathological data indicated that the hypomethylated ETS1 motif may be an effective indicator for high-grade tumor in BRCA1-mutated ovarian cancer. Moreover, univariate survival analysis demonstrated an association between the hypomethylated ETS1 motif and an increased risk of death. This study provides new insights into the causes and prognosis of hypomethylated ETS1 motifrelated PARP1 activation in BRCA1-mutated ovarian cancer. The mechanism involves synergistic effects of promoter methylation, histone modification and transcription factor binding. Therefore, a more specific epigenetic therapy could be developed for BRCA1mutated ovarian cancer.

\section{METHODS}

\section{Ethics Statement}

Investigation has been conducted in accordance with the ethical standards and according to the Declaration of Helsinki and according to national and international guidelines and has been approved by the authors' institutional review board.
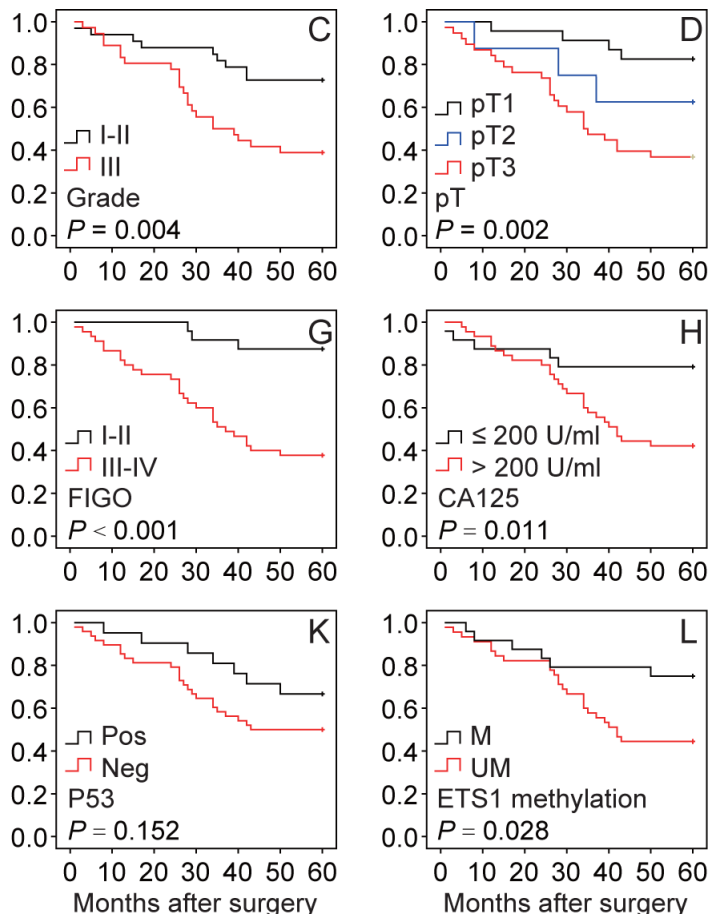

Fig 4: Kaplan-Meier analysis of overall survival for 69 BRCA1-mutated ovarian cancer patients. The following variables were analyzed: age at diagnosis, menstruation, grade, pT, pN, pM, FIGO, CA125, ascites, residual tumor, and p53 and ETS1 methylation. Pre, premenopausal; Post, postmenopausal; FIGO, International Federation of Gynecology and Obstetrics; Pos, positive; Neg, negative; M, methylated; UM, unmethylated. 


\section{Patients and tissue collection}

Serous ovarian cancer patients were enrolled between 2010 and 2012, and all patients gave informed consent. Fresh tumor samples, adjacent normal ovarian tissues, ascites and blood samples were obtained at the time of primary surgery before any chemotherapy or radiotherapy. Hematoxylin and eosin staining of the samples for histopathological diagnosis and grading were determined by three staff pathologists using the World Health Organization criteria. The tumor stages were assessed according to the FIGO and TNM classifications. All patients were screened for BRCA1 mutations by multiplex polymerase chain reaction with complete sequence analysis as previously reported [7]. Their characteristics are given in Table 1.

\section{Cell culture, lentiviral infection and cell proliferation assay}

Primary ovarian cancer cells were obtained from ascites for patients undergoing surgery for ovarian cancer and cultured in RPMI 1640 with $10 \%$ fetal bovine serum (Invitrogen, CA USA) as described previously [16]. Human 293T cells and SKOV3 ovarian cancer cells were maintained in DMEM with $10 \%$ fetal bovine serum (Invitrogen). Lentiviral vectors expressing short hairpin RNAs (shRNA) against BRCA1 (NM 007299) were obtained from GeneChem Co., Ltd (Shanghai, China), and synthesized as follows: Forward: 5-CCGGAACCTGTCTCCACAAAGTG TGCTCGAGCACACTTTGTGGAGACA GGTTT TTTTG,andReverse:5AATTCAAAAAAACCTGTC TCCA CAAAGTGTGCTCGAGCACACTTTGTG GAGACAGGTT. The non-silencing siRNA sequence (TTCTCCGAACGTGTCACGT) was used as a negative control. For overexpression of BRCA1, the open reading frame of BRCA1 (NM 007299) was cloned into the lentiviral vector GV287 (Ubi-MCS-3FLAG-SV40-EGFP) (GeneChem). Transfections were performed using the polybrene and enhanced infection solution (GeneChem) according to the manufacturer's recommended protocol. The shRNA lentiviral particles of GCN5 (sc-37946-V), PCAF (sc-36198-V) and ETS1 (sc-29309-V) were purchased from Santa Cruz Biotechnology (CA, USA). Transfections were performed using the polybrene and enhanced infection solution (GeneChem) according to the manufacturer's recommended protocol. After 48 hours of infection, cell proliferation was determined using the CellLight $^{\mathrm{TM}}$ EdU Apollo®643 In Vitro Imaging Kit (Ribobio, Guangzhou, China) following the instructions provided by the manufacturer.

\section{Real-time PCR}

Real-time PCR was performed as previously described [7]. The specific primer sequences for real-time PCR are listed in Supplementary Table 1.

\section{Chromatin immunoprecipitation (ChIP), site- directed mutagenesis, transfection and dual- luciferase reporter assay}

ChIP, site-directed mutagenesis, transfection and dual-luciferase reporter assays were performed as previously described [17]. The specific primer sequences for site-directed mutagenesis and ChIP are provided in Supplementary Table 1 . The specific antibodies for ChIP are provided in Supplementary Table 2.

\section{Co-immunoprecipitation (CO-IP) and immunoblotting}

CO-IP was performed using an immunoprecipitation kit (Invitrogen) according to the manufacturer's recommended protocol. Then, the cell lysates and immunoprecipitates were analyzed by immunoblotting. The specific antibodies for CO-IP and immunoblotting are provided in Supplementary Table 2.

\section{Circular dichroism (CD) spectra}

The CD spectra were obtained on a Jasco J-810 spectropolarimeter at $25^{\circ} \mathrm{C}$ using a $0.1 \mathrm{~cm}$ path length cell; data were collected with a $2 \mathrm{~nm}$ slit width from 350 to 200 $\mathrm{nm}$ at $0.5 \mathrm{~nm}$ intervals and averaged over three scans. CD experiments were carried out on DNA samples $(5 \propto \mathrm{M})$ using a buffer containing $0.2 \mathrm{M}$ phosphate buffer ( $\mathrm{pH}$ 7.0) in the presence of $100 \mathrm{mM} \mathrm{Na}+$ or $\mathrm{K}+$. The DNA samples were annealed by heating to $95^{\circ} \mathrm{C}$ for $5 \mathrm{~min}$ followed by cooling to room temperature over $10 \mathrm{~h}$ before analysis. The DNA sequence was 5- CGTGGCGTCGGGCTTC "C (CH3 or non-CH3)" GGAGCTTTGGCGGCAGCTA, and was synthesized by Sangon Biotech Ltd (Shanghai, China).

\section{Statistical analysis}

The association between clinicopathological features and ETS1 motif methylation was determined using the Fisher's exact test. Univariate analysis of survival was performed using the Kaplan-Meier method. Multivariate Cox regression analysis was performed to identify the independent prognostic factors for overall survival. The data are presented as mean $\pm \mathrm{SD}$. Statistical differences in the data were evaluated by Student's t test or one-way 
ANOVA as appropriate, and were considered significant at $\mathrm{P}<0.05$.

\section{ACKNOWLEDGEMENTS}

This work was supported by the 973 Program of China (No. 2011CB933504), Natural Science Foundation of China (No. 81071072) and the Higher Specialized Research Fund for Doctoral Program of Ministry of Education of China (No. 20122104110027).

\section{REFERENCES}

1. Lech A, Daneva T, Pashova S, Gagov H, Crayton R, Kukwa W, Czarnecka AM, Szczylik C. Ovarian cancer as a genetic disease. Front Biosci. 2013; 18:543-563.

2. Pruthi S, Gostout BS, Lindor NM. Identification and Management of Women With BRCA Mutations or Hereditary Predisposition for Breast and Ovarian Cancer. Mayo Clin Proc. 2010; 85:1111-1120.

3. Murphy SK. Targeting the epigenome in ovarian cancer. Future Oncol. 2012; 8:151-164.

4. Seeber LM, van Diest PJ. Epigenetics in ovarian cancer. Methods Mol Biol. 2012; 863:253-269.

5. Bryant HE, Schultz N, Thomas HD, Parker KM, Flower D, Lopez E, Kyle S, Meuth M, Curtin NJ, Helleday T. Specific killing of BRCA2-deficient tumours with inhibitors of poly(ADP-ribose) polymerase. Nature. 2005; 434:913-917.

6. Farmer H, McCabe N, Lord CJ, Tutt AN, Johnson DA, Richardson TB, Santarosa M, Dillon KJ, Hickson I, Knights C, Martin NM, Jackson SP, Smith GC, Ashworth A. Targeting the DNA repair defect in BRCA mutant cells as a therapeutic strategy. Nature. 2005; 434:917-921.

7. Bi FF, Li D, Yang Q. Promoter hypomethylation, especially around the E26 transformation-specific motif, and increased expression of poly (ADP-ribose) polymerase 1 in BRCAmutated serous ovarian cancer. BMC Cancer. 2013; 13:90.

8. Bochman ML, Paeschke K, Zakian VA. DNA secondary structures: stability and function of G-quadruplex structures. Nat Rev Genet. 2012; 13:770-780.

9. Suva ML, Riggi N, Bernstein BE. Epigenetic reprogramming in cancer. Science. 2013; 339:1567-1570.

10. Zhang B, Zhou Y, Lin N, Lowdon RF, Hong C, Nagarajan RP, Cheng JB, Li D, Stevens M, Lee HJ, Xing X, Zhou J, Sundaram V, Elliott G, Gu J, Shi T, et al. Functional DNA methylation differences between tissues, cell types, and across individuals discovered using the M\&M algorithm. Genome Res. 2013; 23:1522-1540.

11. Gutierrez-Hartmann A, Duval DL, Bradford AP. ETS transcription factors in endocrine systems. Trends Endocrinol Metab. 2007; 18:150-158.

12. Soldatenkov VA, Albor A, Patel BK, Dreszer R, Dritschilo A, Notario V. Regulation of the human poly(ADP-ribose) polymerase promoter by the ETS transcription factor.
Oncogene. 1999; 18:3954-3962.

13. Yao JJ, Liu Y, Lacorazza HD, Soslow RA, Scandura JM, Nimer SD, Hedvat CV. Tumor promoting properties of the ETS protein MEF in ovarian cancer. Oncogene. 2007; 26:4032-4037.

14. Brenne K, Nymoen DA, Hetland TE, Trope CG, Davidson B. Expression of the Ets transcription factor EHF in serous ovarian carcinoma effusions is a marker of poor survival. Hum Pathol. 2012; 43:496-505.

15. Cao J, Song Y, Bi N, Shen J, Liu W, Fan J, Sun G, Tong T, He J, Shi Y, Zhang X, Lu N, He Y, Zhang H, Ma K, Luo X, et al. DNA methylation-mediated repression of miR-886$3 p$ predicts poor outcome of human small cell lung cancer. Cancer Res. 2013; 73:3326-3335.

16. Szlosarek PW, Grimshaw MJ, Kulbe H, Wilson JL, Wilbanks GD, Burke F, Balkwill FR. Expression and regulation of tumor necrosis factor alpha in normal and malignant ovarian epithelium. Mol Cancer Ther. 2006; 5:382-390.

17. Fan-xin M, Li-mei S, Bei S, Xin Q, Yu Y, Yu C. Heat shock factor 1 regulates the expression of the TRPV1 gene in the rat preoptic-anterior hypothalamus area during lipopolysaccharide-induced fever. Exp Physiol. 2012; 97:730-740. 